\title{
Mechanics of Intact Bone Marrow
}

\author{
Lauren E. Jansen ${ }^{1}$, Nathan P. Birch ${ }^{1}$, Jessica D. Schiffman ${ }^{1}$, Alfred J. Crosby ${ }^{2}$,
} Shelly R. Peyton ${ }^{* 1}$

1. Department of Chemical Engineering, University of Massachusetts Amherst, 686 N. Pleasant St., 159 Goessmann Hall, Amherst, Ma 01003, USA

2. Polymer Science and Engineering Department, University of Massachusetts Amherst, 120

Governors Dr., Amherst, Ma 01003, USA

*Corresponding Author

Shelly R. Peyton

Department of Chemical Engineering

University of Massachusetts Amherst

N531 Life Sciences Laboratories

240 Thatcher Rd

Amherst MA 01003-9364

speyton@ecs.umass.edu

+1.413 .545 .1133 (office)

University of Massachusetts Amherst, MA 01003 


\begin{abstract}
The current knowledge of bone marrow mechanics is limited to its viscous properties, neglecting the elastic contribution of the extracellular matrix. To get a more complete view of the mechanics of marrow, we characterized intact yellow porcine bone marrow using three different, but complementary techniques: rheology, indentation, and cavitation. Our analysis shows that bone marrow is elastic, and has a large amount of intra- and inter-sample heterogeneity, with an effective Young's modulus ranging from $0.25-24.7 \mathrm{kPa}$ at physiological temperature. Each testing method was consistent across matched tissue samples, and each provided unique benefits depending on user needs. We recommend bulk rheology to capture the effects of temperature on tissue elasticity and moduli, indentation for quantifying local tissue heterogeneity, and cavitation rheology for mitigating destructive sample preparation. We anticipate the knowledge of bone marrow elastic properties for building in vitro models will elucidate mechanisms involved in disease progression and regenerative medicine.
\end{abstract}

Key words: cavitation; indentation; rheology; Young's modulus; contact mechanics

\title{
1. Introduction
}

Bone marrow plays a significant role in body homeostasis by regulating immune and stromal cell trafficking. Researchers have characterized the matrix content and the role of local cells in bone physiology, but capturing the mechanics of bone marrow tissue has been limited in scope. The elastic modulus of engineered substrates is well known to influence cell shape, proliferation, migration and differentiation (Marklein and Burdick, 2010; Peyton and Putnam, 2005; Peyton et al., 2008; Yang et al., 2014). While significant effort has gone into recapitulating the hematopoietic microenvironment in vitro for both regenerative medicine and to improve drug screening, there is no physiological measurement of the modulus of intact bone marrow (Lee et 
al., 2012; Mahadik et al., 2014; Nicholsa et al., 2010; Scotti et al., 2013; Torisawa et al., 2014). Though some of these model systems incorporate controlled mechanics, there is little validation for the stiffness choices, even though bone marrow stromal and progenitor cells are mechanically responsive to both engineered substrates, and the viscosity of the surrounding fluid (Engler et al., 2006; Lee et al., 2014; Sikavitsas et al., 2003; Yang et al., 2014). Knowing the modulus of in vivo tissue is critical for regenerative medicine as well. For example, the Blau lab found that the regenerative capacity of muscle stem cells is enhanced when cultured on surfaces mechanically similar to mouse muscle (Gilbert et al., 2011). This highlights the need for methods that can appropriately characterize the heterogeneous mechanics of bone marrow tissue to understand its role in driving the behaviors of the cells within.

Marrow tissue has hematopoietic-rich and adipose-rich regions, which are referred to as red and yellow marrow, respectively. Yellow marrow is enriched in the medullary cavity and red marrow in the spongy, trabecular bone (Parfitt et al., 1983; Vande Berg et al., 1998). Cell content in the marrow is a dynamic process, and yellow marrow can expand and contract as haematopoiesis occurs (Gimble et al., 1996). Unfortunately, the difficulty of harvesting red marrow has limited the ability to isolate and test its mechanics using conventional methods. Yellow marrow has been shown to be mechanically heterogeneous in studies where samples are homogenized and centrifuged to remove cell and bone debris (Bryant, 1988; Zhong and Akkus, 2011). Prepping samples in this manner removes many of the inconsistencies caused when harvesting marrow, but ignores the elastic contribution of the bone marrow extracellular. The most robust study on yellow marrow mechanics measured the viscosity of the marrow from 19 human subjects and found no apparent correlation between age and marrow viscosity, though marrow has been shown to yellow with age (Justesen et al., 2001; Zhong and Akkus, 2011). 
Another group found proximal bovine marrow, the tissue close to the trabecular bone, to be more viscous than distal bovine marrow, and they suggest that these changes in viscosity are a function of spatial marrow composition (Bryant et al., 1988). Though both of these studies are informative, the impact of the surrounding or, potentially inclusive, trabecular bone is neglected because samples were homogenized and filtered.

The anatomical location and surrounding cortical bone poses a unique challenge for researchers interested in mechanically studying bone marrow tissue. Many studies have looked at properties of homogenized marrow, by extracting marrow from the medullary cavity and performing bulk rheology, but these approaches are destructive and create a critical gap in our knowledge of intact marrow mechanics (Bryant, 1988; Bryant et al., 1988; Saito et al., 2002; Sobotkova et al., 1988; Zhong and Akkus, 2011). Additionally, researchers have used techniques to measure intramedullary pressure (IMP) to better understand how lifestyle choices, such as loading, disuse, steroid use, and diseases such as osteoporosis and cancer change marrow content, blood flow, and bone remodeling (Bloomfield, 2010; Gurkan and Akkus, 2008; Lynch et al., 2013; Miyanishi et al., 2002; Zhang et al., 2007). It is clear that many external factors impact IMP changes, but no work has gone into characterizing the mechanics of the intact matrix, which we suggest plays a stiffness-dependent role in disease progression. Rheology is the dominant method used to characterize bone marrow tissue, with the exception of one group that used ultrasonic wave propagation (Hosokawa and Otani, 1997). However, ultrasonic wave propagation reported that the Young's modulus of bovine marrow is the same order of magnitude as what others have found for the surrounding spongy bone ( 2GPa) (Morgan et al., 2003). The stark differences between marrow and bone likely make it hard to distinguish the marrow mechanics with type of technique. Though the viscoelasticity of bone marrow makes it ideal for 
bulk rheological characterization, this technique lacks the ability to measure microscopic level heterogeneities and often requires destructive sample preparation. Since bone marrow tissue varies across the length of the bone, is cell-rich, and is highly vascularized, we aimed to explore two more sensitive methods in parallel with traditional rheology: indentation and cavitation rheology. This approach enabled us to explore the continuity of diverse mechanical techniques and sample preparations on the characterization of marrow mechanical properties. Together, this information will allow the field of tissue engineering to further improve the understanding of marrow mechanics and to build more accurate in vitro models of marrow tissue.

\section{Materials and Methods}

\subsection{In vitro sample preparation}

Femurs from grass-fed large black Tamworth Cross pigs, 6-10 months old, were gathered from a local butcher, and mechanical testing was conducted within $2 \mathrm{hrs}$ post-opening of the bone cavity. Indentation and rheology samples were gathered from a bone cut lengthwise down the femur, and tissue samples were biopsy punched out of the medullary cavity and stored in phosphate buffer solution ( $\mathrm{pH}$ 7.4) for mechanical testing (Figure 1a). The porcine bones were cut horizontally across the medullary cavity of a femur for cavitation rheology testing. All indentation and cavitation tests were performed at room temperature, which ranges from 18$22^{\circ} \mathrm{C}$ and is annotated as $20^{\circ} \mathrm{C}$ throughout the paper. A minimum of 6 porcine bones, from different pigs, were used for each mechanical test.

\subsection{Rheology}

Small amplitude oscillatory shear measurements were performed in a Kinexus Pro rheometer (Malvern Instruments, UK) using a plate-plate geometry, with a diameter of $20 \mathrm{~mm}$ and gap of 1 $\mathrm{mm}$. Porcine bone marrow punches were placed on the lower plate, the top plate was lowered into position, and excess marrow was trimmed with a razor blade. A solvent trap was placed over the geometry and temperature was maintained at $25^{\circ} \mathrm{C}$. A $0.15 \%$ strain was selected from a strain amplitude sweep to ensure that experiments were conducted within the linear viscoelastic region 
(Suppl. Figure 1). Oscillatory frequency sweeps were conducted between 0.1 and $16 \mathrm{~Hz}$. To capture temperature variation, samples were heated to $35^{\circ} \mathrm{C}$, and the measurements were repeated. The effective Young's modulus, $E^{E f f}$, was calculated at a frequency of $0.1 \mathrm{~Hz}$ assuming a Poisson's ratio, $v$, of 0.5 .

$E^{E f f}=2 G^{*}(1+v)$

\subsection{Indentation}

Indentation is a custom-built instrument that measures the forces applied to materials mounted on a stage (Chan et al., 2008). A flat, cylindrical steel probe (High-Speed M2 Tool Steel Hardened Undersized Rod, $5.2 \mathrm{~mm}$ diameter) was brought into contact with the tissue sample. The test was carried out at a fixed displacement rate $(10 \mu \mathrm{m} / \mathrm{s})$, and the maximum applied relative displacement was $100 \mu \mathrm{m}$. The force, $P$, was monitored by a force transducer (Honeywell Sensotec, Columbus, $\mathrm{OH}$ ) connected in series with a nanoposition manipulator (Burleigh Instruments Inchworm Model IW-820) that controlled the displacement, $\delta$. A customdeveloped National Instruments LabVIEW code recorded the material compliance, $C$, which is the change in displacement over the change in force:

$$
c=\frac{d \delta}{d P}
$$

Applying a correction ratio to account for the dimensional confinement described by the ratio between the contact radius, $a$, to the sample height, $h(0.5<a / h<2)$, the effective Young's

modulus can be determined from the measured compliance by (Shull et al., 1998):

$$
E^{E f f}=\frac{3}{8 C\left\{1+1.33(a / h)+1.33(a / h)^{3}\right\} a}
$$

\subsection{Cavitation rheology}

All measurements were taken using a custom-built instrument that consists of a syringe pump (New Era Syringe Pump NE1000), pressure sensor (Omega Engineering PX26-001GV), and 
syringe needle connected to a DAQ card that records pressure as a function of time. The needle is inserted into the tissue sample $(2-5 \mathrm{~mm})$ and air is injected at a rate of $5000 \mu \mathrm{L} / \mathrm{min}$. The pressure is recorded using a custom-developed LabVIEW code until the air cavity in the tissue bursts. Three measurements, in separate tissue regions, were taken for a range of needle gauges (16-32 gauge, 0.838-0.108 mm). Pressure of cavitation is a function of needle radius, and an effective Young's modulus can be calculated with the following equation assuming an isotropic material on the size scale of the needle (Hutchens and Crosby, 2014):

$$
P_{c}=1.05 E^{E f f}+\frac{2.1 \gamma}{r}
$$

where $P_{c}$ is the pressure of cavitation, $\gamma$ is surface tension, and $r$ is the inner radius of the needle.

\subsection{Statistical Analysis}

Statistical analysis was accomplished using Graphpad's Prism v5.0a. Data are reported as mean \pm standard error. Statistical significance was determined by a one-way analysis of variance (ANOVA) followed by a Tukey's multiple comparisons test. When noted, a two-tailed t-test was used. P-values $<0.05$ are considered significant, where $\mathrm{p}<0.05$ is denoted with $*, \leq 0.01$ with $* *$, $\leq 0.001$ with $* * *$, and $\leq 0.0001$ with $* * * *$.

\section{Results}

\subsection{Three techniques were used to characterize the elastic modulus of bone marrow}

We harvested intact marrow, with minimal post-mortem time, from 6-10 month old pigs. Pig was chosen as a model organism because of their anatomical similarity to humans, and their widespread use as sources for biological materials and as subjects for medical device testing (Meurens et al., 2012; Sullivan et al., 2001). Further, the pig model allowed us to examine tissue-scale mechanical heterogeneity absent of many convoluting factors, such as age, diet, and race. Rheology, indentation, and cavitation were used to mechanically characterize bone marrow 
tissue samples. Rheology and indentation samples were prepared by removing biopsy punches from the inner medullary cavity, and cavitation rheology was done using the horizontal crosssection of the femur as a site for needle insertion (Figure 1a). Rheology measures the bulk properties of a biopsy punch placed between two oscillating parallel plates (Figure 1b). Indentation, an axisymmetric compression test, is a micro-scale measurement with a flat-punch probe that is an order of magnitude smaller than the parallel plates used in rheology (Figure 1c). Indentation measures the substrate load as a function of time and displacement, allowing the use of basic principles of contact mechanics to calculate an effective modulus. Cavitation rheology is also a micro-scale measurement, and is performed by flowing a solvent, in this case air, into a substrate, which creates an inner cavity (Figure 1d). After the cavity bursts at the cavitation pressure, the Young's modulus can be calculated from Equation 4 using the surface tension between the substrate, solvent, and inner radius of the cavitation needle.

\subsection{Bone marrow is a benign tissue with dominant elastic contributions}

We used bulk rheology to quantify the viscoelastic properties of bone marrow tissue. The dynamic moduli are frequency dependent, and the dynamic storage modulus is consistently an order of magnitude larger than the dynamic loss modulus (Figure 2a). Rheology was the only instrument with temperature control, and, as the temperature was increased to $35^{\circ} \mathrm{C}$, both the dynamic moduli and complex viscosity decreased by an order of magnitude. The complex viscosity decreased as shear rate increased, indicating that bone marrow behaves as a nonNewtonian fluid (Figure 2b). Power law indices of $0.15 \pm 0.01$ for $25^{\circ} \mathrm{C}$ and $0.12 \pm 0.02$ for $35^{\circ} \mathrm{C}$ determined that bone marrow is a benign material with dominant elastic contributions to its mechanical response, which becomes slightly increased elastic at higher temperatures. This 
behavior was mirrored in indentation, which showed that marrow closely followed a Hertzian model upon compression (Suppl. Figure 2a, c-d).

\subsection{Porcine bone marrow has inter- and intra-sample heterogeneity}

At $25^{\circ} \mathrm{C}$, the effective Young's moduli for marrow ranged from $0.73-135.6 \mathrm{kPa}$ for rheology and 0.42-64.5 kPa for indentation (Figure 3a-b, Suppl. Table 1). Instrument sensitivity was gauged using intra-sample standard deviation, which showed that indentation was slightly better than rheology at capturing the spatial heterogeneity within each sample. At physiological temperature $\left(35^{\circ} \mathrm{C}\right)$, the effective Young's moduli for marrow ranged from 0.1-10.9 $\mathrm{kPa}$ (Suppl. Figure 3). Indentation validated that the intra-sample heterogeneity was a function of spatial modulus differences within the samples, and not an effect of the instrument damaging the tissue sample (Suppl. Figure 2b). At the tested frequencies, both rheology and indentation consistently reported similar effective Young's moduli for matched bone samples. To connect the spatial differences to marrow location in the medullary cavity, we separated the marrow into proximal and distal sections. There was no significant difference in effective Young's modulus between the distal and proximal marrow samples from the same bone (Suppl. Figure 4). Surprisingly, there was no trend in harvest location in the cavity and sample modulus, suggesting that marrow location in the medullary cavity is not the cause of the apparent intra-sample heterogeneity.

\subsection{Sample removal affects the modulus of marrow}

Cavitation rheology is the least destructive technique for sample preparation, enabling us to assess the modulus of marrow within the hard, osseous bone cavity. The pressure of cavitation is a function of needle radius, allowing an effective Young's modulus to be calculated for a nonelastic material (Figure 4a). The effective Young's modulus for marrow ranged from 8.5-64.3 $\mathrm{kPa}$, and we found that these values were consistent with the variation seen in the matched bones 
for rheology and indentation (Figure 4B). Two of the bone samples (5 and 6) did not cavitate prior to reaching the maximum limit for the pressure sensor, therefore an effective Young's modulus could not be inferred. These samples were the stiffest measured by both rheology and indentation, which likely explains the observed instrument limitation.

\subsection{Continuity across mechanical methods}

We found a strong continuity between all the mechanical tests and the estimated effective Young's modulus (Table 1). All the methods fluctuated at the same magnitude for matched bone samples, validating the use of these instruments for material characterization. While the matched values calculated for rheology and indentation were indistinguishable from one another (Spearmen correlation of rheology vs. indentation, $\rho=1.0, * *)$, cavitation consistently reported a higher modulus for each of the bone samples (Spearmen correlation of rheology vs indentation and rheology, $\rho=0.4$, n.s.).

\section{Discussion}

A goal of tissue engineering is to recapitulate key features of tissues in vitro in order to better understand in vivo phenomena and apply this toward directing tissue function. The mechanical properties of a cell's microenvironment have been shown to dictate the migration and differentiation of marrow-derived mesenchymal stem cells, but little research has been conducted on mechanically characterizing bone marrow tissue (Engler et al., 2006; Peyton et al., 2011; Yang et al., 2014). To fully capture the heterogeneity of intact bone marrow, we present and propose three complementary methods, with the intention of highlighting some of the advantages and disadvantages of each method for mechanical testing. Here, we report that bone marrow is a benign viscoelastic tissue with a dominant elastic contribution and significant intra- and intersample heterogeneity across three mechanical testing methods. 
Our data for bone marrow mechanics is both much stiffer, and encompasses a larger range of values than earlier studies. However, as previously noted, other studies have been performed on homogenized tissue samples, so the reported viscosities ranging from of 44.6-142 $\mathrm{mPa} \cdot \mathrm{s}$ cannot be easily compared to our viscous data (range from 100-500 Pa.s) (Bryant, 1988; Bryant et al., 1988; Saito et al., 2002; Sobotkova et al., 1988; Zhong and Akkus, 2011). Intact bone marrow tissue has an effective Young's modulus ranging from $0.25-24.7 \mathrm{kPa}$ at physiological marrow temperature $\left(35^{\circ} \mathrm{C}\right.$, Petrakis, 1952) (Suppl. Figure 2, Suppl. Table 1). The only other report of rheology on intact marrow found that bovine marrow has a dynamic storage modulus of $\sim 220 \mathrm{~Pa}$ at a frequency of $1.6 \mathrm{~Hz}$ and temperature of $37^{\circ} \mathrm{C}$ (Winer et al., 2009). At this same frequency, but at $35^{\circ} \mathrm{C}$, our porcine samples had a dynamic storage modulus ranging from 23-10,000 $\mathrm{Pa}$ (data not shown). This study is consistent with the storage magnitude we report for intact marrow, but because their project was confined to 3 samples from the same bone, this limited their ability to capture biological heterogeneities in marrow samples. We found intact marrow to have a large amount of inter-sample heterogeneity, and this is not surprising because biological tissues are known to be heterogeneous (Figure 3). For example, reports on the elastic modulus of brain and lung tissue can range from $0.1-10$, and $1.5-100 \mathrm{kPa}$, respectively (Booth et al., 2012; Chatelin et al., 2010; Lai-Fook and Hyatt, 2000; Melo et al., 2014; Miller et al., 2000; Rashid et al., 2013; Zhong and Akkus, 2011). While it is more likely that these variations are due to structural components of the tissues, it was also important to validate that the array of mechanical tests used to gather these values were not the source of this heterogeneity, as we have done here.

There is opposing literature on how tissue mechanics change in response to any processing post-mortem. One group showed no effect on viscosity after freezing marrow tissue 
samples; however, another group discovered that any post-processing (i.e. freezing or postmortem time) dramatically changes the elastic modulus of brain tissue (Davis and Praveen, 2006; Rashid et al., 2013). Cavitation was the least destructive of the three techniques, making it ideally suited to mitigate post-mortem processing of tissue samples. Cavitation captured the same magnitude changes in samples as rheology and indentation, but consistently reported a higher modulus. This implies that removing the marrow from the medullary cavity could be lowering the tissue modulus. It is possible that the biopsy punch compromised tension formed from protein fibrils and vasculature in bone marrow tissue, which would explain the lower reported modulus for the other methods. Alternatively, the spongy nature of bone marrow tissue could cause the sudden drop in pressure to be associated with the onset of a fracture event, rather than the initiation of an elastic cavitation event (Kundu and Crosby, 2009). If fracture occurs prior to cavitation, then this also implies that Equation 4 is not valid for bone marrow tissue. The tissue opacity and surrounding cortical bone makes the latter case difficult to assess, and a more thorough study is needed to investigate cavity propagation in marrow tissue. Regardless, future studies should consider the possibility that sample preparation may compromise the modulus of bone marrow tissue.

We predicted that indentation would be more sensitive to local heterogeneities than rheology, because the probe size is an order of magnitude smaller and allows for control of probe location. Contrary to our initial thoughts, indentation provided only a slight sensitivity advantage (Figure 3). This suggests that the heterogeneities contributing to changes in bone marrow modulus are on a size scale smaller than indentation can capture. We speculate that a technique like atomic force microscopy (AFM) may be able to improve our ability to annotate these 
modulus driven heterogeneities, if the sample preparation for this technique is made to be less destructive.

We recommend conventional rheology for samples where temperature control is needed (Suppl. Figure 3). Though the effective modulus of porcine marrow was strongly temperaturedependent, the tissue remained with a dominant elastic contribution at all temperatures tested (i.e. power law held at $\sim 0.15$ ). This suggests that cavitation and indentation are still valid methods for certain measurements, even though they are performed at room temperature (Figure 2b). If modulus is not a major function of temperature, indentation provides speed, simplicity of use, and reports the same metrics as rheology. We observed that indentation was slightly better at capturing spatial heterogeneity when directly compared with bulk rheology, and adjusting to a smaller probe size could improve this spatial characterization (Figure 2). Cavitation was the only method that allowed for in situ testing in the bone cavity. A possible challenge in cavitation is the need to know the substrate-fluid surface tension to convert pressure of cavitation $\left(P_{c}\right)$ to an effective Young's modulus $\left(E^{E f f}\right.$ (Figure 4A). We avoided this by using multiple needle gauges to interpolate an effective Young's modulus. However, if cavitation rather than fracture can be confirmed, then at large needle sizes only one simple measurement is required because $P_{c}=E^{*} 1.05$ (Hutchens and Crosby, 2014). This could provide significant advantages, but more fundamental research on detecting the differences between cavitation and fracture is required (Hutchens and Crosby, 2014; Kundu and Crosby, 2009). We suggest cavitation is the most appropriate technique for testing tissue samples in situ, but methods to avoid breaking the endosteal membrane or causing tissue trauma need to be refined for this technique to be effective. The three methods reported similar values for modulus, making them all valid ways to calculate an elastic modulus for a soft material. 
It is interesting that the observed tissue heterogeneity could not be parsed out through annotation of tissue location, or by changing the magnitude of the probe compressing the tissue (Suppl. Figure 4). We used indentation and rheology at annotated locations within a bone sample in an attempt to connect modulus with marrow location. Cell content differentiates red and yellow bone marrow, but the anatomical challenge of harvesting pure red-bone marrow limited us to using the marrow adjacent to the trabecular bone, where hematopoietic cells are enriched (Bryant et al., 1988; Vande Berg et al., 1997). We found no significant difference in the effective Young's modulus between proximal and distal marrow samples (Suppl. Figure 4). These finding only emerge when including the extracellular matrix contribution, and contradict previous studies on homogenized tissues showing proximal bovine marrow has a higher viscosity than distal marrow (Bryant, 1988). This study also found that the removal of cell debris and granular matter from homogenized tissue decreased the fluid viscosity, suggesting that these factors contribute to the dynamic loss modulus. Though the reason for the apparent heterogeneity is unclear, this study, alongside our results, suggest that cell content may have little impact on the storage modulus, and we advise future studies tolook into how the matrix protein content relates to local modulus changes in intact marrow.

\section{Conclusion}

Here, we used three non-destructive approaches to mechanically characterize intact bone marrow. Across rheology, indentation, and cavitation we found that bone marrow is a benign viscoelastic material. Also, we are the first to report that marrow has dominant elastic contribution when the tissue is intact, and we feel this knowledge supports that components of the microenvironment, besides blood flow, may contribute to body homeostasis is a stiffness dependent manner. We also stress that bone marrow tissue is heterogeneous, and there is not one, 
but a range of appropriate moduli values for marrow. Overall, this type of thorough

characterization can be used to improve upon current studies of bone marrow tissue elasticity and gain new insights into tissue function and structure.

\section{Acknowledgements}

We are grateful to Michael Imburgia, Dr. Sami Fakhouri, and Shruti Rattan for technical assistance and for insightful conversations. We would also like to thank Lauren Barney, Elizabeth Brooks, Sualyneth Galarza, and Dr. Sam Polio for useful advice in manuscript preparation. SRP is a Pew Biomedical Scholar supported by the Pew Charitable Trusts. SRP was supported by a faculty development award from Barry and Afsaneh Siadat. This work was funded by an NIH New Innovator award (1DP2CA186573-01) awarded to SRP, a grant from the NSF to AJC (DMR-1304724), and start-up funds from the University of Massachusetts Amherst. NPB and JDS thank the James M. Douglas Career Development Faculty Award for support.

\section{References}

Bloomfield, S. a., 2010. Disuse osteopenia. Curr. Osteoporos. Rep. 8, 91-97. doi:10.1007/s11914-010-0013-4

Booth, A.J., Hadley, R., Cornett, A.M., Dreffs, A. a., Matthes, S. a., Tsui, J.L., Weiss, K., Horowitz, J.C., Fiore, V.F., Barker, T.H., Moore, B.B., Martinez, F.J., Niklason, L.E., White, E.S., 2012. Acellular normal and fibrotic human lung matrices as a culture system for in vitro investigation. Am. J. Respir. Crit. Care Med. 186, 866-876. doi:10.1164/rccm.201204-0754OC

Bryant, J., 1988. On the mechanical function of marrow in long bones. Eng. Med. 17, 55-58.

Bryant, J., David, T., Gaskell, P., King, S., Lond, G., 1988. Rheology of bovine bone Marrow. Proc Instn Mech Engrs 203, 71-75.

Chan, E.P., Smith, E.J., Hayward, R.C., Crosby, A.J., 2008. Surface Wrinkles for Smart Adhesion. Adv. Mater. 20, 711-716. doi:10.1002/adma.200701530 
Chatelin, S., Constantinesco, A., Willinger, R., 2010. Fifty years of brain tissue mechanical testing: From in vitro to in vivo investigations. Biorheology 47, 255-276. doi:10.3233/BIR2010-0576

Davis, B.L., Praveen, S., 2006. Nonlinear versus linear behaviour of calcaneal bone marrow at different shear rates, in: Annual Meeting of American Society of Biomaterials.

Engler, A.J., Sen, S., Sweeney, H.L., Discher, D.E., 2006. Matrix elasticity directs stem cell lineage specification. Cell 126, 677-89. doi:10.1016/j.cell.2006.06.044

Gilbert, P., Havenstrite, K., Magnusson, K., Sacco, A., Leonardi, N., Kraft, P., Nguyen, N., Thrun, S., Lutolf, M., Blau, H., 2011. Substrate elasticity regulates skeletal muscle stem cell self- renewal in culture. Science (80-. ). 329, 1078-1081.

doi:10.1126/science.1191035.Substrate

Gimble, J.M., Robinson, C.E., Wu, X., Kelly, K. a., 1996. The function of adipocytes in the bone marrow stroma: An update. Bone 19, 421-428. doi:10.1016/S8756-3282(96)00258-X

Gurkan, U.A., Akkus, O., 2008. The mechanical environment of bone marrow: a review. Ann. Biomed. Eng. 36, 1978-91. doi:10.1007/s10439-008-9577-x

Hosokawa, A., Otani, T., 1997. Ultrasonic wave propagation in bovine cancellous bone. J. Acoust. Soc. Am. 101, 558-62.

Hutchens, S.B., Crosby, A.J., 2014. Soft-solid deformation mechanics at the tip of an embedded needle. Soft Matter 10, 3679-84. doi:10.1039/c3sm52689e

Justesen, J., Stenderup, K., Ebbesen, E.N., Mosekilde, L., Steiniche, T., Kassem, M., 2001. Adipocyte tissue volume in bone marrow is increased with aging and in patients with osteoporosis. Biogerontology 2, 165-171.

Kundu, S., Crosby, A.J., 2009. Cavitation and fracture behavior of polyacrylamide hydrogels. Soft Matter 5, 3963. doi:10.1039/b909237d

Lai-Fook, S.J., Hyatt, R.E., 2000. Effects of age on elastic moduli of human lungs. J. Appl. Physiol. 89, 163-168.

Lee, J., Abdeen, A. a, Kilian, K. a, 2014. Rewiring mesenchymal stem cell lineage specification by switching the biophysical microenvironment. Sci. Rep. 4, 5188. doi:10.1038/srep05188

Lee, J., Li, M., Milwid, J., Dunham, J., Vinegoni, C., Gorbatov, R., Iwamoto, Y., Wang, F., Shen, K., Hatfieeld, K., Enger, M., Shafiee, S., McCormack, E., Ebert, B.L., Weissleder, R., Yarmush, M.L., Parekkadan, B., 2012. Implantable microenvironments to attract hematopoietic stem / cancer cells. Proc. Natl. Acad. Sci. 109, 19638-19643. doi:10.1073/pnas.1208384109//DCSupplemental.www.pnas.org/cgi/doi/10.1073/pnas.1208384109 
Lynch, M.E., Brooks, D., Mohanan, S., Lee, M.J., Polamraju, P., Dent, K., Bonassar, L.J., Van Der Meulen, M.C.H., Fischbach, C., 2013. In vivo tibial compression decreases osteolysis and tumor formation in a human metastatic breast cancer model. J. Bone Miner. Res. 28, 2357-2367. doi:10.1002/jbmr.1966

Mahadik, B.P., Wheeler, T.D., Skertich, L.J., Kenis, P.J., Harley, B.C., 2014. Microfluidic generation of gradient hydrogels to modulate hematopoietic stem cell culture environment. Adv. Healthc. Mater. 3, 449-58. doi:10.1002/adhm.201300263

Marklein, R. a., Burdick, J. a., 2010. Spatially controlled hydrogel mechanics to modulate stem cell interactions. Soft Matter 6, 136. doi:10.1039/b916933d

Melo, E., Cárdenes, N., Garreta, E., Luque, T., Rojas, M., Navajas, D., Farré, R., 2014. Inhomogeneity of local stiffness in the extracellular matrix scaffold of fibrotic mouse lungs. J. Mech. Behav. Biomed. Mater. 37, 186-195. doi:10.1016/j.jmbbm.2014.05.019

Meurens, F., Summerfield, A., Nauwynck, H., Saif, L., Gerdts, V., 2012. The pig: A model for human infectious diseases. Trends Microbiol. 20, 50-57. doi:10.1016/j.tim.2011.11.002

Miller, K., Chinzei, K., Orssengo, G., Bednarz, P., 2000. Mechanical properties of brain tissue in-vivo $\square$ : experiment and computer simulation 33, 1369-1376.

Miyanishi, K., Yamamoto, T., Irisa, T., Yamashita, a., Jingushi, S., Noguchi, Y., Iwamoto, Y., 2002. Bone marrow fat cell enlargement and a rise in intraosseous pressure in steroidtreated rabbits with osteonecrosis. Bone 30, 185-190. doi:10.1016/S8756-3282(01)00663-9

Morgan, E.F., Bayraktar, H.H., Keaveny, T.M., 2003. Trabecular bone modulus-density relationships depend on anatomic site. J. Biomech. 36, 897-904. doi:10.1016/S00219290(03)00071-X

Nicholsa, J.E., Cortiellab, J., Leee, J., Nilesa, J.A., Cuddihy, M., Wangg, S., Cantua, A., Mlcakb, R., Valdiviaa, E., Yancy, R., Bielitzkii, J., McClurea, M.L., Kotov, N.A., 2010. In vitro analog of human bone marrow from 3D scaffolds with biomimetic inverted colloidal crystal geometry. Biomaterials 30, 1071-1079. doi:10.1016/j.biomaterials.2008.10.041.In

Parfitt, A., Mathews, C., Villanueva, A., Kleerekoper, M., 1983. Relationships between Surface, Volume, and Thickness of Iliac Trabecular Bone in Aging and in Osteoporosis. J. Clin. Invest. 72, 1396-1409.

Petrakis, N.L., 1952. Temperature of Human Bone Marrow. J. Appl. Physiol. 4, 549-553.

Peyton, S.R., Kalcioglu, Z.I., Cohen, J.C., Runkle, A.P., Van Vliet, K.J., Lauffenburger, D.A., Griffith, L.G., 2011. Marrow-derived stem cell motility in 3D synthetic scaffold is governed by geometry along with adhesivity and stiffness. Biotechnol. Bioeng. 108, 1181-93. doi:10.1002/bit.23027 
Peyton, S.R., Kim, P.D., Ghajar, C.M., Seliktar, D., Putnam, A.J., 2008. The effects of matrix stiffness and RhoA on the phenotypic plasticity of smooth muscle cells in a 3-D biosynthetic hydrogel system. Biomaterials 29, 2597-2607. doi:10.1016/j.biomaterials.2008.02.005

Peyton, S.R., Putnam, A.J., 2005. Extracellular Matrix Rigidity Governs Smooth Muscle Cell Motility in a Biphasic Fashion. J. Cell. Physiol. 209, 198-209. doi:10.1002/jcp.20274

Rashid, B., Destrade, M., Gilchrist, M.D., 2013. Influence of preservation temperature on the measured mechanical properties of brain tissue. J. Biomech. 46, 1276-1281.

Saito, H., Lai, J., Rogers, R., Doerschuk, C.M., 2002. Mechanical properties of rat bone marrow and circulating neutrophils and their responses to inflammatory mediators. Blood 99, 220713.

Scotti, C., Piccinini, E., Takizawa, H., Todorov, A., Bourgine, P., Papadimitropoulos, A., Barbero, A., Manz, M.G., Martin, I., 2013. Engineering of a functional bone organ through endochondral ossification. Proc. Natl. Acad. Sci. U. S. A. 110, 3997-4002. doi:10.1073/pnas.1220108110

Shull, K.R., Ahn, D., Chen, W.-L., Flanigan, C.M., Crosby, A.J., 1998. Axisymmetric adhesion test of soft materiasl. Macromol. Chem. Phys. 199, 489-511.

Sikavitsas, V.I., Bancroft, G.N., Holtorf, H.L., Jansen, J. a, Mikos, A.G., 2003. Mineralized matrix deposition by marrow stromal osteoblasts in 3D perfusion culture increases with increasing fluid shear forces. Proc. Natl. Acad. Sci. U. S. A. 100, 14683-14688. doi:10.1073/pnas.2434367100

Sobotkova, E., Hruba, A., Kiefman, J., Sobotka, Z., 1988. Rhelogocal behavior of bone marrow. Biorheology 467-469.

Sullivan, T.P., Eaglstein, W.H., Davis, S.C., Mertz, P., 2001. The pig as a model for human wound healing. Wound Repair Regen. 9, 66-76.

Torisawa, Y.-S., Spina, C.S., Mammoto, T., Mammoto, A., Weaver, J.C., Tat, T., Collins, J.J., Ingber, D.E., 2014. Bone marrow-on-a-chip replicates hematopoietic niche physiology in vitro. Nat. Methods 11, 663-9. doi:10.1038/nmeth.2938

Vande Berg, B.C., Lecouvet, F.E., Moysan, P., Maldague, B., Jamart, J., Malghem, J., 1997. MR assessment of red marrow distribution and composition in the proximal femur: Correlation with clinical and laboratory parameters. Skeletal Radiol. 26, 589-596. doi: $10.1007 / \mathrm{s} 002560050291$

Vande Berg, B.C., Malghem, J., Lecouvet, F.E., Maldague, B., 1998. Magnetic resonance imaging of normal bone marrow. Eur. Radiol. 8, 1327-1334. doi:10.1007/s003300050547 
Winer, J., Janmey, P. a., McCormick, M., Funaki, M., 2009. Bone Marrow-Derived Human Mesenchymal Stem Cells Become Quiescent on Soft Substrates but Remain Responsive to the Chemical or Mechanical Stimuli. Tissue Eng. Part A 15, 147-154.

Yang, C., Tibbitt, M.W., Basta, L., Anseth, K.S., 2014. Mechanical memory and dosing influence stem cell fate. Nat. Mater. 13, 645-52. doi:10.1038/nmat3889

Zhang, P., Su, M., Liu, Y., Hsu, A., Yokota, H., 2007. Knee loading dynamically alters intramedullary pressure in mouse femora. Bone 40, 538-543. doi:10.1016/j.bone.2006.09.018

Zhong, Z., Akkus, O., 2011. Effects of age and shear rate on the rheological properties of human yellow bone marrow. Biorheology 48, 89-97. doi:10.3233/BIR-2011-0587

Figure 1. Techniques used to characterize porcine bone marrow. a: Samples were removed from the medullary cavity of femurs for rheology and indentation (left). The horizontal crosssection of the femur was used as the needle insertion point for cavitation (right). b: Rheology measurements were done between two parallel plates to obtain a storage $\left(G^{\prime}\right)$ and loss $\left(G^{\prime \prime}\right)$ modulus with respect to increasing shear. c: Indentation is an axisymmetric compression test that records the load (shown) and displacement over time. d: Cavitation records pressure as a cavity is propagated in a substrate over time. The formation of this cavity is a function of inner needle radius and solvent-substrate surface tension. The point when the cavity collapses is recorded as the pressure of the cavitation $\left(P_{c}\right)$.

Figure 2. Rheological behavior of porcine marrow. a: Representative rheological data. The storage $\left(G^{\prime}\right.$, open symbol) and loss $\left(G^{\prime}\right.$, closed symbol) modulus versus strain rate show a weakly frequency dependent material with a dominant elastic modulus. As temperature is increased from $25^{\circ} \mathrm{C}$ (blue) to $35^{\circ} \mathrm{C}$ (red), both modulus and b: complex viscosity decrease, indicating strong temperature dependence. The power law $(\alpha)$ fit of the complex viscosity showed that bone marrow is slightly more elastic at the higher temperature (t-test, $* \mathrm{p}<0.05)$. 
Figure 3. Bone marrow exhibits inter- and intra- sample heterogeneity. The effective Young's modulus $\left(E^{E f f}\right)$ for a: rheology $\left(0.1 \mathrm{~Hz}\right.$ at $\left.25^{\circ} \mathrm{C}\right)$ and $\mathbf{b}$ : indentation $\left(0.03 \mathrm{~Hz}\right.$ at $\left.20^{\circ} \mathrm{C}\right)$ in matched bone samples shows strong continuity between measurements from both instruments. Data points represent different locations within the same bone sample. Statistical significance was calculated using the mean of the data $(* \mathrm{P}<0.05$, $* * \mathrm{P}<0.01, * * * \mathrm{P}<0.001)$.

Figure 4. Cavitation mirrors indentation and rheology measurements. a: The pressure of cavitation $\left(P_{c}\right)$ changes as a function of needle radius, $r$, allowing an effective Young's modulus $\left(E^{E f f}\right)$ to be derived using Equation 4 and the y-intercept (red). The inset is a picture of the bone cross-sectional area, which is the site of needle insertion. b: The $E^{E f f}$ at $20^{\circ} \mathrm{C}$ is similar to the modulus calculated for matched tissue samples in rheology and indentation. The solid line represents no data (n.d.) because these samples maxed out the instrument pressure sensor. 
Table I. Comparison of effective Young's Modulus obtained from in vitro methods for samples from the same bone.

\begin{tabular}{cccc}
\hline $\begin{array}{c}\text { Marrow } \\
\text { Sample }\end{array}$ & $\begin{array}{c}\text { Rheology } \\
(\mathrm{kPa})\end{array}$ & $\begin{array}{c}\text { Indentation } \\
(\mathrm{kPa})\end{array}$ & $\begin{array}{c}\text { Cavitation } \\
(\mathrm{kPa})\end{array}$ \\
\hline Temperature & $25^{\circ} \mathrm{C}$ & $20^{\circ} \mathrm{C}$ & $20^{\circ} \mathrm{C}$ \\
\hline 1 & $52.1 \pm 10.2$ & $30.3 \pm 4.0$ & $64.3 \pm 0.2$ \\
2 & $4.0 \pm 0.9$ & $5.7 \pm 0.3$ & $9.0 \pm 0.01$ \\
3 & $0.7 \pm 0.3$ & $0.9 \pm 0.2$ & $8.5 \pm 3.7$ \\
4 & $3.2 \pm 1.9$ & $2.1 \pm 0.3$ & $14.4 \pm 10.0$ \\
5 & $84.4 \pm 6.5$ & $35.3 \pm 4.9$ & no data \\
6 & $135.6 \pm 25.6$ & $37.1 \pm 6.3$ & no data \\
7 & $69.0 \pm 21.4$ & --- & --- \\
8 & --- & $12.2 \pm 2.8$ & --- \\
9 & --- & --- & $16.0 \pm 1.6$ \\
\hline
\end{tabular}


- Bone marrow is a benign material, that exhibits a large amount of intra- and inter-tissue heterogeneity

- Intact porcine marrow has an effective Young's modulus ranging from $0.25-24.7 \mathrm{kPa}$ at physiological temperature

- Bulk rheology is the best method to capture the effects of temperature on tissue viscoelasticity

- Indentation is more sensitive than rheology, making it the ideal method for quantifying local tissue heterogeneity

- Cavitation rheology mitigates destructive sample preparation, so tissue mechanics can be tested in situ 

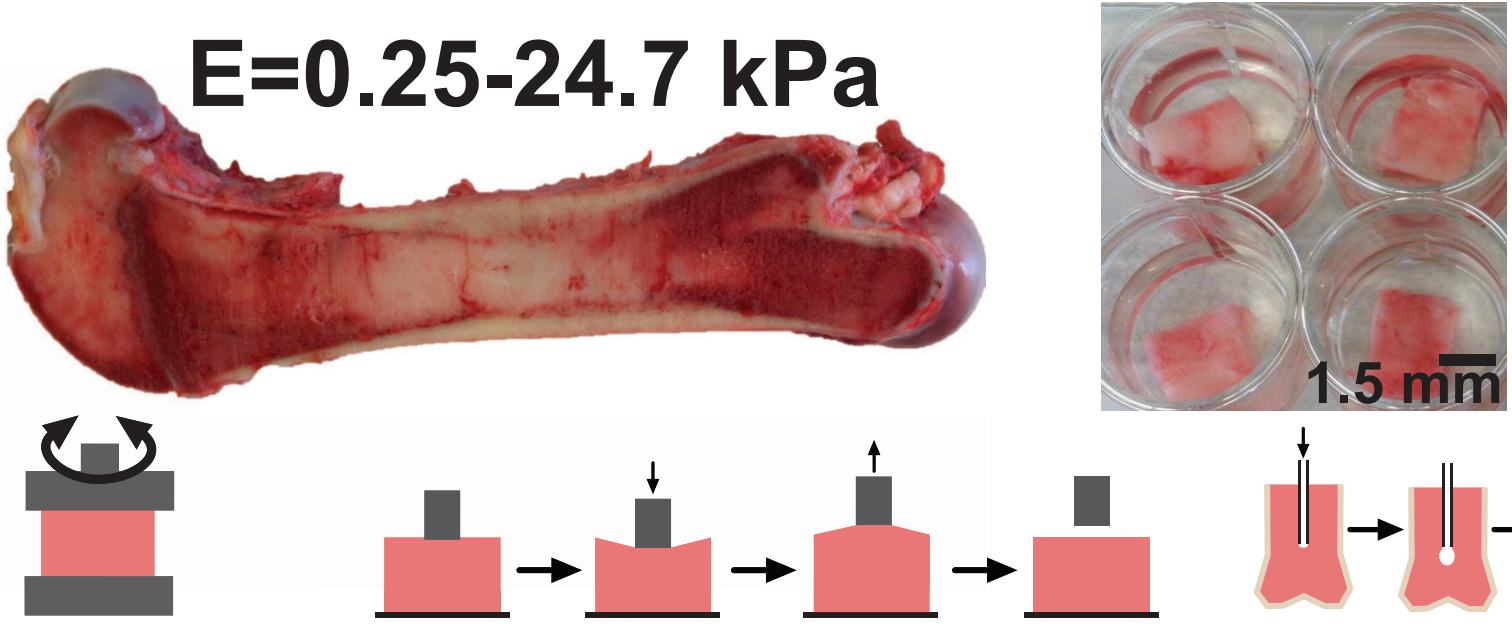

$\stackrel{t}{\|} \cdot \mid \|$ Rheology

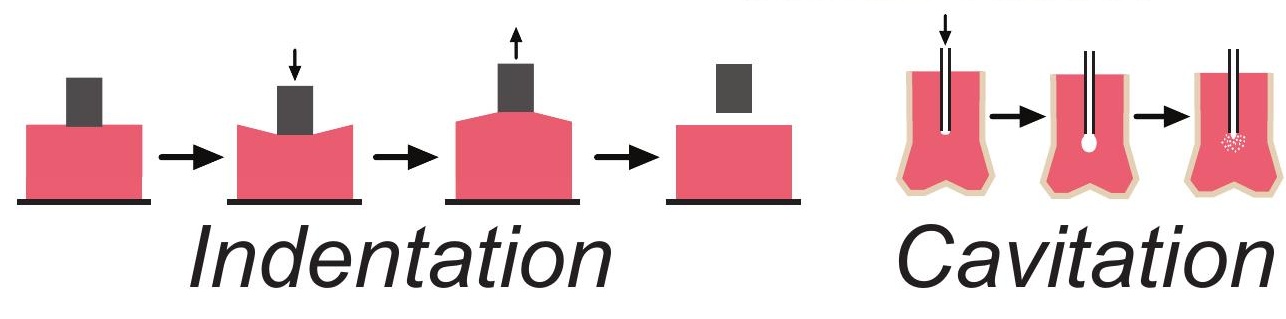

Cavitation 


\section{Porcine Bone}

a Marrow Samples

Rheology and

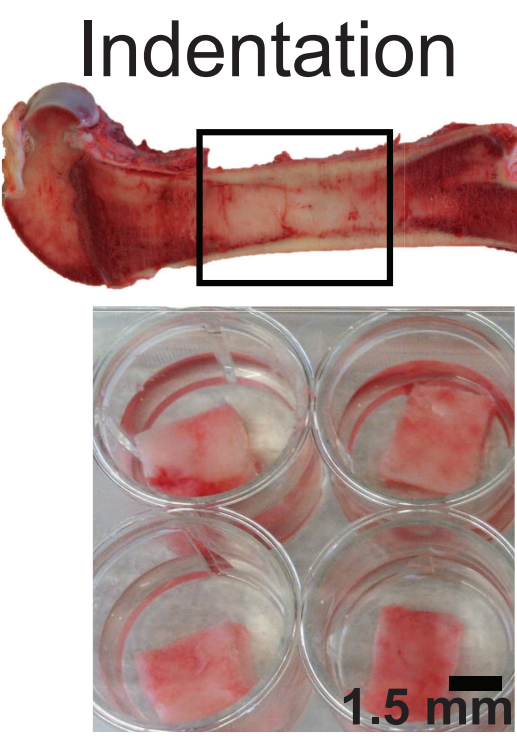

Cavitation

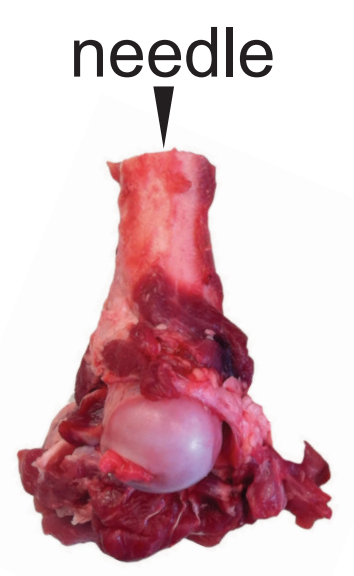

C Indentation
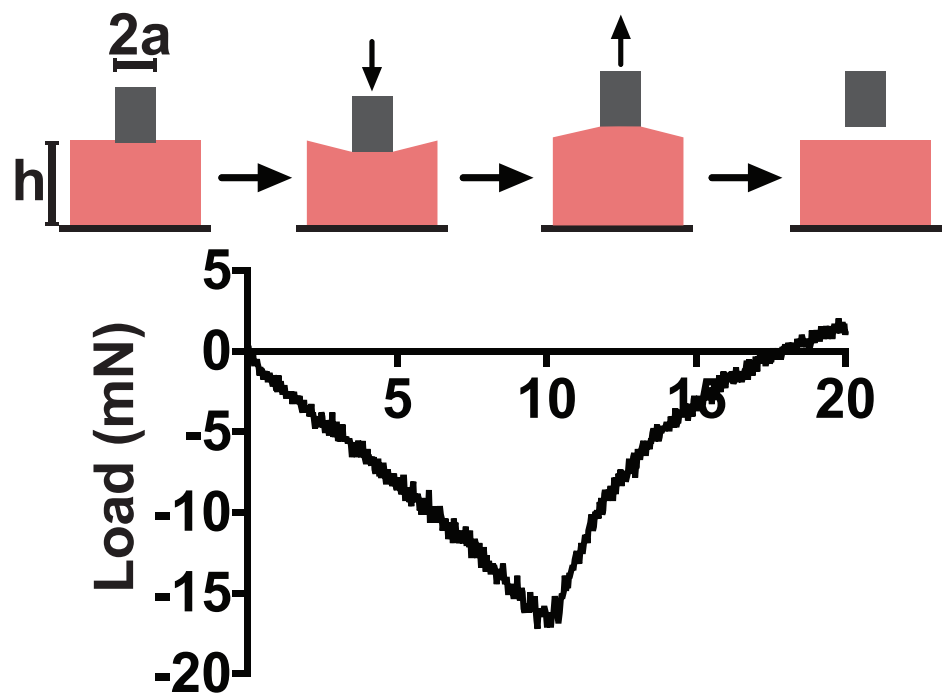

Time (s) b Rheology

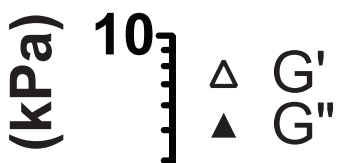

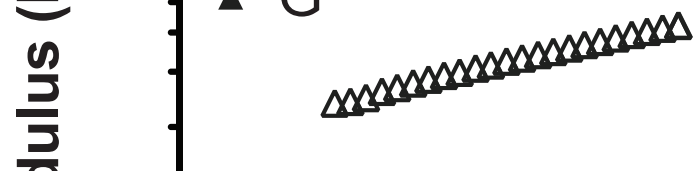

$\begin{array}{llll}0.1 & & & \\ 0.01 & 0.1 & 1 & 10\end{array}$

$\omega\left(\mathbf{s}^{-1}\right)$

d

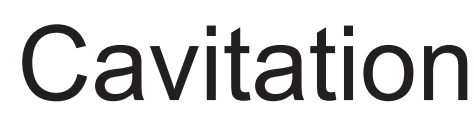
$\downarrow$ Solvent Pc

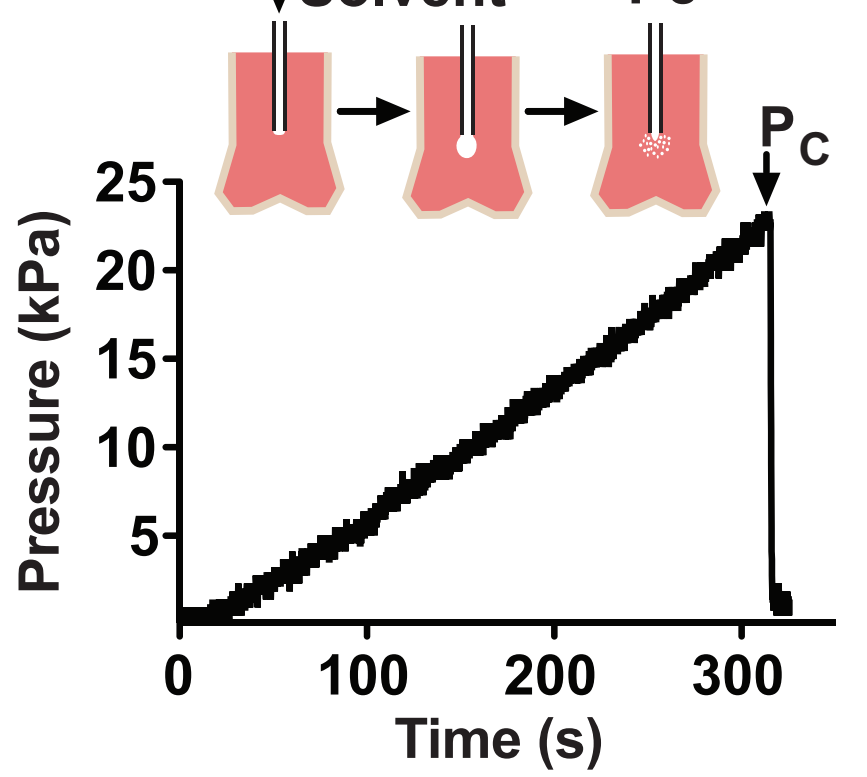




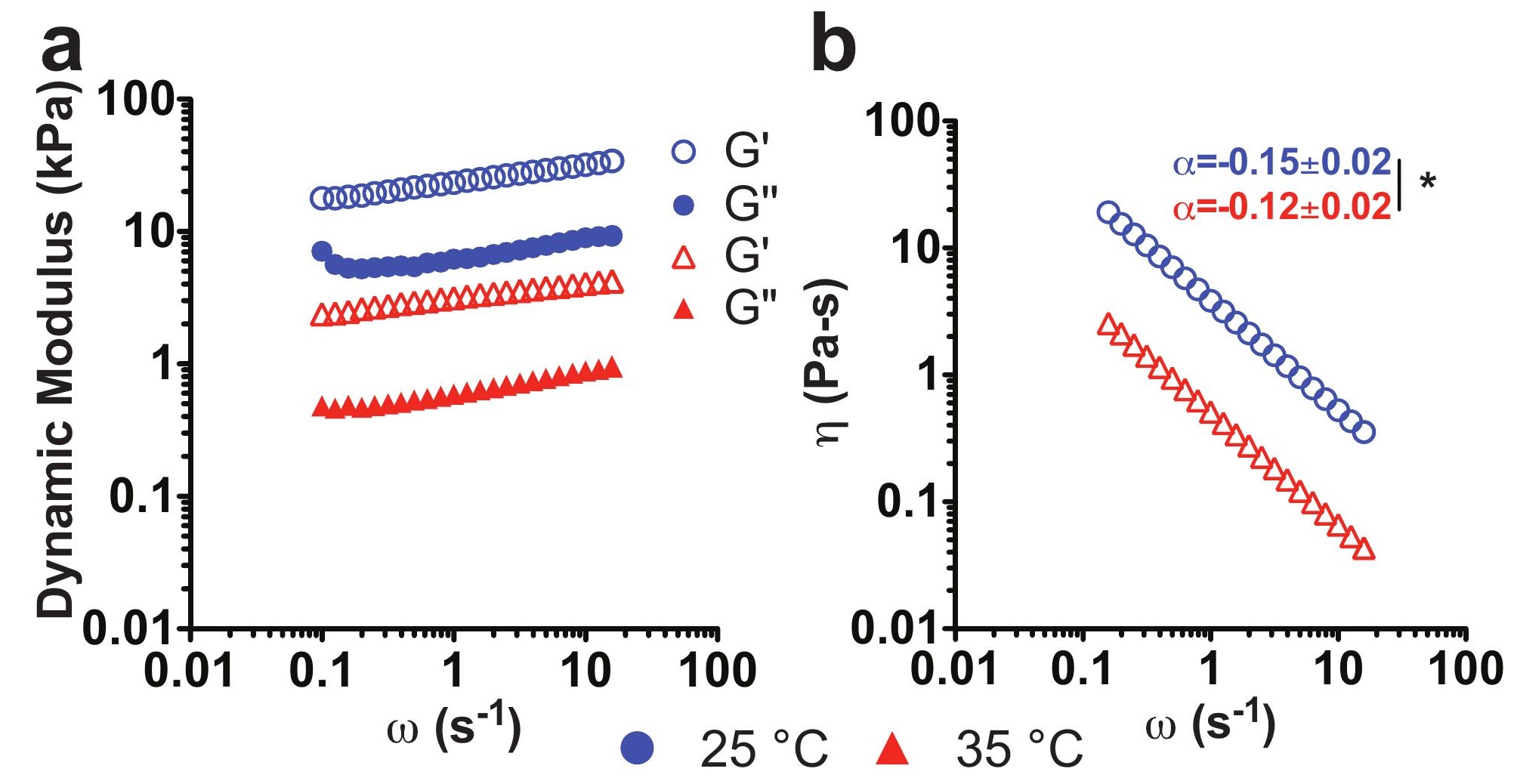

Figure 2 
Figure 3

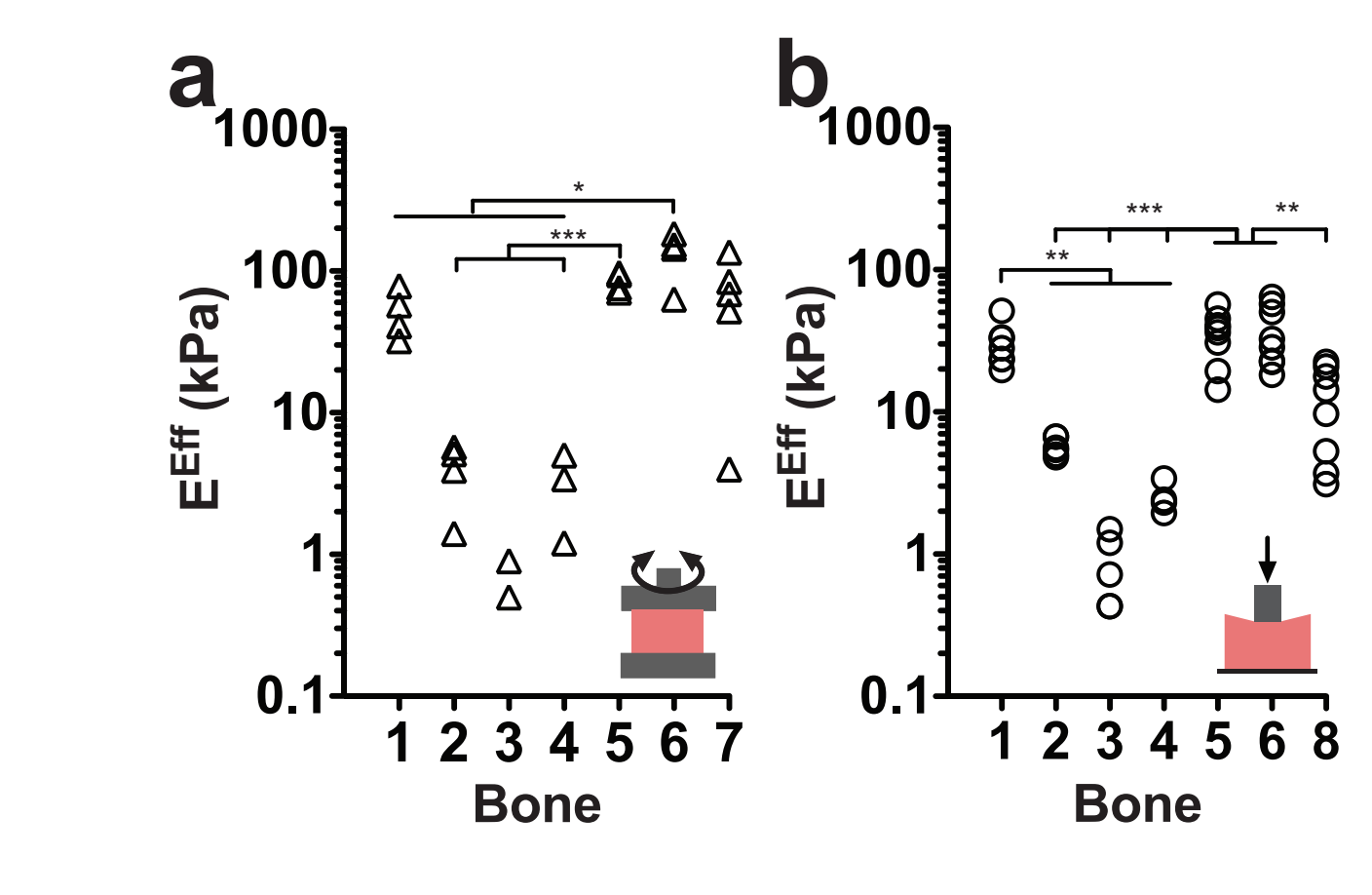



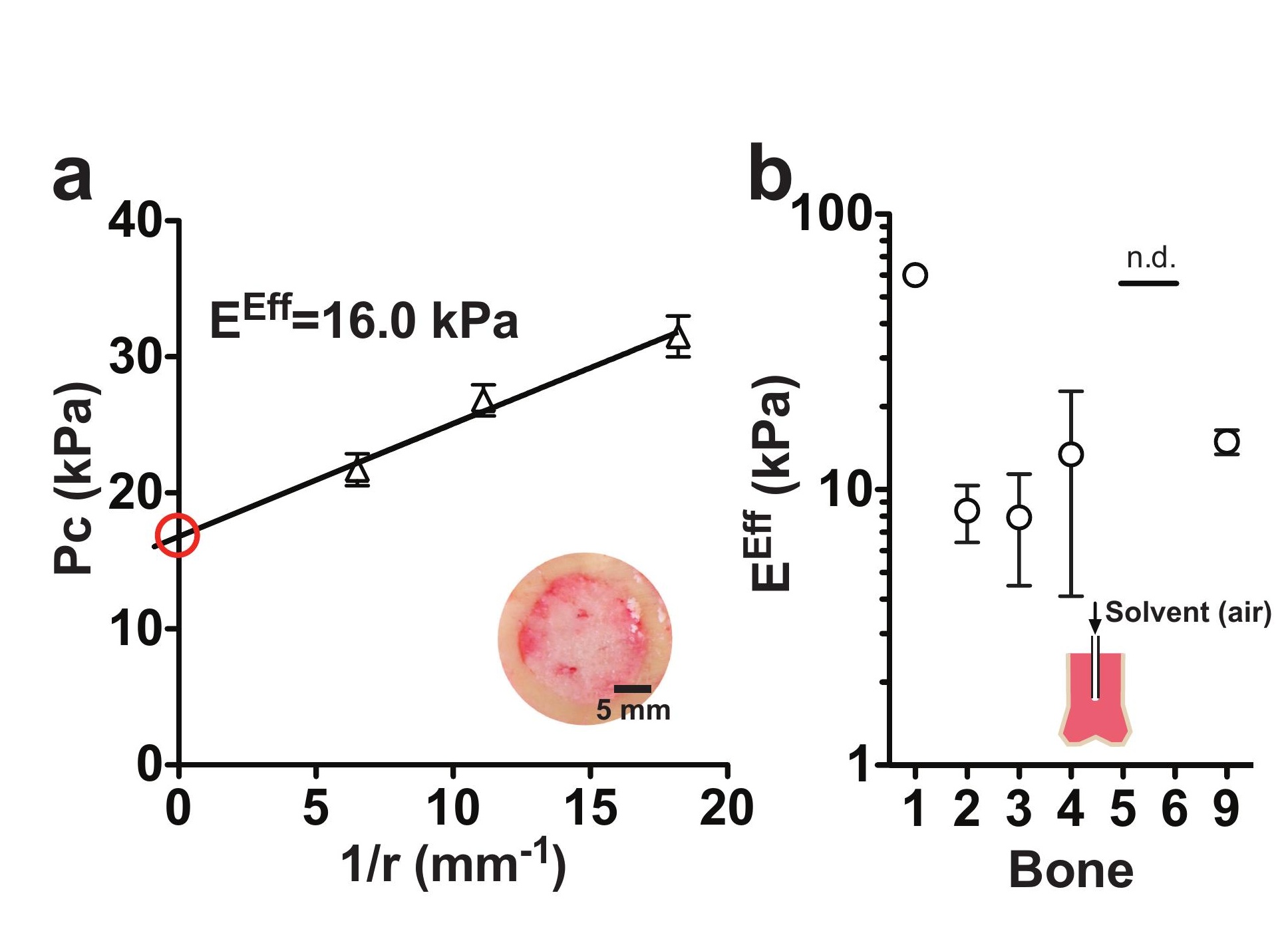

Figure 4

\section{(1)}

4

